ERRATUM

doi:10.1038/nature25499

\section{Erratum: Non-adaptive plasticity potentiates rapid adaptive evolution of gene expression in nature}

Cameron K. Ghalambor, Kim L. Hoke, Emily W. Ruell, Eva K. Fischer, David N. Reznick \& Kimberly A. Hughes

Nature 525, 372-375 (2015); doi:10.1038/nature15256

There are several errors in this Letter. First, owing to a production error, the Sequence Reads Archive (SRA) accession number is published as 'SRP06236' rather than 'SRP062364'. Second, owing to an author error, the sample size for the number of samples for the low-predation (LP) population reared without predators is listed in the Methods as ' $n=3$ ', rather than ' $n=4$ '. Third, owing to an author error, in Supplementary Table 1, the treatment columns referred to as 'NP' and ' $\mathrm{P}$ ' are reversed for the HP population. Furthermore, we have identified an error in the code used to produce some of the simulations. Permutation distributions used to identify concordantly differentially expressed (CDE) genes inadvertently omitted data from the LP population. We have now reanalysed the data, including that population. In this revised analysis, all of the originally reported patterns remain unchanged, but the magnitude of statistical significance is reduced compared to the original analysis. The main results and conclusions remain unchanged, but because the reanalysis alters the set of genes deemed CDE, all subsequent tests based on the subset of CDE genes also required reanalysis. The changes to the original text, tables and figures reported in this Letter are described in more detail below.

(1) The number of adaptively evolved (CDE) genes decreased from 125 to 94 , and remains outside the interquartile range for the permutated distribution $(23,73)$, and in the upper decile of the permuted distribution.

(2) The correlation between evolutionary change and non-adaptive plasticity (Fig. 2 of the original Letter) remains almost unchanged $(-0.81$ in the new analysis, and -0.82 in the original analysis).
As before, the new correlation remains well outside the interquartile range of the permuted correlations $(-0.47,-0.59)$, and smaller than any of the 1,000 permutation values. See Supplementary Fig. 1 to this Erratum.

(3) The correlation between ancestral and derived plasticity (Fig. 3 of this Letter) has increased from -0.588 in the original analysis to -0.743 in the new analysis. As before, the new correlation remains well outside the interquartile range of the permuted correlations $(-0.57,-0.68)$, and in the lower $3.5 \%$ of the permuted values. See Supplementary Fig. 2 to this Erratum.

(4) The results comparing the observed amount of divergence in gene expression versus the amount of putative neutral divergence reported has remained almost unchanged (Extended Data Table 1 of the original Letter). The new $P_{\mathrm{ST}}$ values for 'Intro1' and 'Intro2' are 0.33 and 0.25 versus the original $P_{\mathrm{ST}}$ values of 0.32 and 0.27 , respectively. All other results remain the same. Supplementary Table 1 to this Erratum contains the corrected version of the original Extended Data Table 1 that shows the comparison of gene expression divergence $\left(P_{\mathrm{ST}}\right)$ with divergence of putatively neutral microsatellite loci $\left(F_{\mathrm{ST}}\right)$.

(5) The number of significantly plastic genes increased from 565 to 658 (in Supplementary Table 2 and Extended Data Fig. 4 of the original Letter). As before, most of these transcripts exhibit plastic changes within the ancestral population that are in the opposite direction of population divergence: $67 \%$ (439 out of 658 ) versus $75 \%$ (424 out of 565 ) in the original analysis. The observation of 658 significantly plastic genes is well outside the interquartile range and in the lower $4 \%$ of permuted values. See Supplementary Fig. 3 to this Erratum.

The permutation tests used in this study are critical in determining whether the observed patterns lie outside the range of expected values given the structure of the data. The inclusion of the naturally occurring LP population in the reanalysis presented here represents a more conservative test of the occurrence of adaptively evolved CDE genes, and as a result weakens the magnitude-but not the direction-of some of the observed patterns. In summary, support for the main results and conclusions remains robust and our interpretation of the data remains unchanged. The original Letter has not been corrected online.

Supplementary Information is available in the online version of this Erratum. 